\title{
BMP7 Ameliorated Viral Myocardial Fibrosis by Inhibiting Endothelial-to-Mesenchymal Transition
}

\section{Yeqing Xie}

Zhongshan Hospital

Jianquan Liao

Department of Cardiology, Zhongshan Hospital, Fudan University, Shanghai 200032هChina

\section{Yong Yu}

Key Laboratory of Viral Heart Diseases, Ministry of Public Health囚Shanghai Institute of Cardiovascular

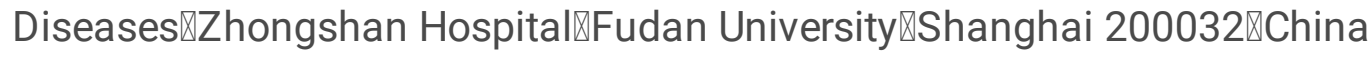

Ruizhen Chen ( $\square$ chenruizhen2020@126.com)

Department of Cardiology, Zhongshan Hospital, Fudan University, Shanghai 200032هChina

https://orcid.org/0000-0002-4449-0449

\section{Xiaoqiang Ding}

Division of Nephrology, Zhongshan Hospital, Fudan University, Shanghai 200032هChina

\section{Research}

Keywords: Viral myocarditis, endothelial-to-mesenchymal transition, cardiac fibrosis, BMP7, Wnt/ $\beta$ catenin, TGF- $\beta 1 /$ Smads

Posted Date: December 28th, 2020

DOI: https://doi.org/10.21203/rs.3.rs-132246/v1

License: (c) (1) This work is licensed under a Creative Commons Attribution 4.0 International License.

Read Full License 
BMP7 ameliorated viral myocardial fibrosis by inhibiting endothelial-to-mesenchymal transition

Yeqing Xie ${ }^{1 *}$, Jianquan Liao ${ }^{2 *}$, Yong $\mathrm{Yu}^{3}$, Ruizhen Chen ${ }^{2,3 \#}$, Xiaoqiang Ding ${ }^{1 \#}$

1 Division of Nephrology, Zhongshan Hospital, Fudan University, Shanghai 200032, China

2 Department of Cardiology, Zhongshan Hospital, Fudan University, Shanghai 200032, China

3 Key Laboratory of Viral Heart Diseases, Ministry of Public Health, Shanghai Institute of

Cardiovascular Diseases, Zhongshan Hospital, Fudan University, Shanghai 200032, China

* The authors contributed equally to this article. ${ }^{\#}$ Co-corresponding author: Ruizhen Chen, Email:

chen.ruizhen@zs-hospital.sh.cn; Xiaoqiang Ding, Email: ding.xiaoqiang@zs-hospital.sh.cn

Tel: +8621 6404 1990x2745

Fax: +862164437078 
Abstract:

Background: Endothelial-to-mesenchymal transition (Endo-MT) is associated with myocardial fibrosis in dilated cardiomyopathy (DCM). Endothelial-to-mesenchymal transition (Endo-MT) is induced by coxsackievirus B3 (CVB3) in cardiac microvascular endothelial cells (CMVECs). Bone morphogenetic protein 7 (BMP7) significantly inhibits Endo-MT and the progression of cardiac fibrosis. The study was aimed to investigate the effect and the underlying mechanism of BMP7 on Endo-MT in myocardial fibrosis induce by CVB3 infection in vivo.

Methods: BALB/c mice were intraperitoneally injected by CVB3 to induce viral myocarditis (VMC). Mice were treated with BMP7 after CVB3 infection. Subsequently, all groups of mice were determined by echocardiography, histopathologic and molecular detection.

Results: We found that the ratio of BMP7/TGF- $\beta 1$ in mRNA levels was decreased obviously at different time points after CVB3 injection. BMP7 facilitated the recovery of cardiac function after CVB3 infection via inhibition of myocardial damage, collagen deposition. Double immunofluorescence staining indicated that Endo-MT was implicated in CVB3-induced myocardial fibrosis, which was attenuated by BMP7. The protein levels of pSmad3 and Smad4 were significantly upregulated in VMC group, as well as Wnt/ $\beta$-catenin and the transcription factor snail. BMP7 treatment reversed the changes of these protein levels. Moreover, CO-IP demonstrated the crosstalk between $\beta$-catenin and Smad3 in VMC mice, which was downregulated by BMP7 treatment.

Conclusions: These results indicated that BMP7 obviously ameliorated myocardial fibrosis in CVB3-infected mice via Endo-MT, which was involved in the TGF- $\beta /$ Smad and Wnt $/ \beta$-catenin pathway. $\beta$-Catenin/Smad3 interaction may be associated with Endo-MT in the development of viral myocardial fibrosis.

Keywords: Viral myocarditis; endothelial-to-mesenchymal transition; cardiac fibrosis; BMP7; Wnt/ $\beta$-catenin; TGF- $\beta 1 /$ Smads

\section{Background}

Myocarditis is an inflammatory disease associated with a large variety of triggers, including infectious agents, autoimmune diseases and drugs. Coxsackievirus B3 (CVB3) is still considered to represent the dominant etiological agent in viral myocarditis (VMC). One-third of patients with 
VMC evolve into a chronic stage of disease and progress to dilated cardiomyopathy (DCM) and heart failure, a frequent reason for heart transplantation[1]. Accumulating studies have demonstrated that myocardial fibrosis orchestrates predominantly the development from VMC to DCM[2-4]. The emergence of cardiac fibroblasts with an endothelial cell origin, referred to as endothelial-tomesenchymal transition (Endo-MT), is associated with myocardial fibrosis in DCM[5]. Endo-MT is characterized by the loss of endothelial cell markers (CD31 and VE-cadherin) and acquisition of a mesenchymal phenotype ( a -smooth muscle actin [ a -SMA], fibroblast specific protein 1 [FSP-1])[6, 7]. To date, Endo-MT has emerged as a player in the pathogenesis of tissue fibrosis in various mouse models, including pressure overloaded [7], myocardial infarction [8], diabetes cardiomyopathy [9], dilated cardiomyopathy [5] and aging [10]. Furthermore, our data indicated that Endo-MT was induced by CVB3 infection in cardiac microvascular endothelial cells (CMVECs) in vitro[11]. Therefore, we assume that Endo-MT may contribute to the myocardial fibrosis in CVB3-infected mice.

Bone morphogenetic protein 7 (BMP7), a member of the transforming growth factor- $\beta$ (TGF$\beta)$ superfamily, has opposing effects on many pathophysiological processes[12]. BMP7 counteracts TGF- $\beta$-induced accumulation of myofibroblasts and extracellular matrix (ECM), referred to as an antifibrotic factor[13, 14]. Of note, BMP7 alleviates myocardial fibrosis via inhibition of Endo-MT in the mouse models of pressure overload and chronic allograft rejection [7]. On the other hand, TGF- $\beta$ promotes the proliferation and activation of resident fibroblast and stimulates Endo-MT[7]. However, the role of BMP7 in viral myocarditis is still unclear.

Base on the previous finding, we hypothesize that BMP7 may reduce myocardial fibrosis induced by CVB3 via inhibition of Endo-MT. In this study, we investigate the effect of BMP7 on Endo-MT in myocardial fibrosis induce by CVB3.

\section{Materials and methods}

\subsection{Virus}

CVB3 (Nancy strain) was prepared as previously mentioned with final infection dose of $10^{3}$ $\mathrm{TCID} 50 / \mathrm{ml}[4,11]$

2.2 Mice model of viral myocarditis

All animal procedures were based on the agreement of the Animal Care and Use Committee 
and the Guideline of Animal Experiments of Fudan University. Male BALB/c mice at 4-5 weeks of age were purchased from Fudan University Laboratory Animal Center (Shanghai, China). BALB/c mice were randomly divided into control group $(\mathrm{n}=10)$, VMC group $(\mathrm{n}=20)$, and BMP7 treatment group ( 3 months, $n=20$ ). Mice were intraperitoneally injected by $10^{3}$ TCID50 CVB3 virus to induce Viral myocarditis (VMC group) at day 0. BMP7 $(0.1 \mathrm{ng} / \mathrm{ml}$, alzet, micro-osmotic pump 1002) was continuously administrated to mice by subcutaneously insertion of micro-osmotic pump (model 1002, Durect Corporation) one day before virus injection [15]. All three groups of mice were subjected to ultrasonic examination, myocardial histopathologic and molecular detection by the end of experiment.

\subsection{Echocardiography}

Ultrasonic examination was performed using a VisualSonics Vevo 770 ultrasound imaging system (VisualSonic inc., Toronto, Canada). Mice was anesthetized by isoflurane and more than five cardiac cycles were recorded. Left ventricular end-diastolic dimension (LVEDd), end-systolic dimension (LVEDs), left ventricular ejection fraction (LVEF) and fractional shortening (FS) were then measured.

2.4 Quantitative Reverse Transcription PCR (qRT-PCR)

Total RNA was extracted from heart samples by TRIzol reagent (Invitrogen). cDNA was reverse-transcribed using a commercial kit for one step qRT-PCR (TaKaRa). Virus RNA replication was also determined by qRT-PCR. The primers were as followed: BMP7: 5'-

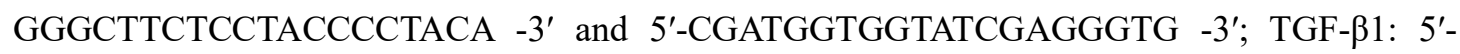
AGGGCTACCATGCCAACTTC-3' and 5'- CCACGTAGTAGACGATGGGC-3'; CVB3: 5'CGGTACCTTTGTGCGCCTGT-3'and 5'-CAGGCCGCCAACGCAGCC-3'[11]; GAPDH (5'TGTGAACGGATTTGGCCGTA -3' and 5'- ACTGTGCCGTTGAATTTGCC-3'). GAPDH mRNA served as a control.

2.5 Histopathological analysis

Cardiac specimens were fixed to make $4 \mu \mathrm{m}$-thick paraffin sections, which were used for hematoxylin-eosin (HE) and Sirius Red (SR) staining in order to describe the characteristics of myofibrils and collagen distribution. Immunohistochemistry was performed with standard protocols for FSP-1 (ab27957, abcam, 1:100) and CD31 (ab24950, abcam, 1:1000). Each sample slice was photographed for at least 10 random chosen high-power fields with Olympus BX51 microscope and 
assessed semi-quantitatively using image-Pro Plus 6.0 analyzing software.

2.6 Double immunofluorescence staining

Confocal fluorescence microscopy was used to explicit the co-location of endothelial markers and mesenchymal markers. $5 \mu \mathrm{m}$-thick frozen heart sections were treated with two mixed primary antibodies at $4^{\circ} \mathrm{C}$ overnight. The primary antibodies groups were respectively $\alpha \mathrm{SMA}(\mathrm{ab} 5694$, rabbit polyclonal, 1:100) and CD31 antibody (ab24950, mouse polyclonal, 1:100), as well as FSP-1 (ab27957, rabbit polyclonal, 1:50) and VE-cadherin antibody (ab7047, mouse polyclonal; 1:50). After washing for at least 3 times, the sections were continually incubated with a compound of two secondary antibodies from different species with conjugation to two distinct fluochromes (respectively goat-anti-rabbit conjugation with rhod-red and goat-anti-mouse conjugation with FITC, Jackson). After slides were stained with DAPI, confocal images were taken (Leica confocal microscope) and the expression of double-labelled picture was analyzed (LAS software).

2.7 Western blot analysis

Western Blot Analysis was performed according to standard procedures. In brief, heart tissues were extracted with RIPA buffer. Protein lysate was separated and transferred to polyvinylidene fluoride membrane, which were incubated with primary antibodies as follows: p-Smad3 (SC11769, 1:500, Santa Cruz Biotechnology), Smad3 (C9513s, 1:1000, Cell Signaling Technology), Smad4 (SC7966, 1:1000, Santa Cruz Biotechnology), Wnt (BS1777, 1:500, Bioworld), $\beta$-catenin (BS3730, 1:500, Bioworld) and snail (BS1853, 1:500, Bioworld). GAPDH was used as an internal standard protein. Secondary antibodies conjugated with HRP (1:1000) were then incubated for 1 hour at room temperature. Finally, band intensity of each sample was assessed using a Quantity One software (Bio-Rad, Hercules, CA, USA).

2.8 Co-immunoprecipitation (Co-IP) assay

Co-IP was performed following standard procedure. Supernatants obtained from heart samples were incubated with selective antibodies and protein $\mathrm{A} / \mathrm{G}$ agarose beads overnight. Beads were washed at least five times and the cleared supernatants were subjected to SDS-PAGE followed by western blot analysis, which was performed with antibodies as mentioned above.

\subsection{Statistics}

SPSS 20.0 was used for data analysis. The one-way analysis of variance (ANOVA) was performed for statistical comparison. $P<0.05$ was considered as statistical significance. 


\section{Results}

3.1 BMP7 improved Cardiac function and reduced fibrosis in viral myocarditis

In the study, we analyzed the effects of BMP7 administration in a mouse model of CVB3induced myocarditis. Inbred male BALB/c mice were injected intraperitoneally with $10^{3} \mathrm{TCID}_{50}$ of CVB3 to establish animal model of viral myocarditis. As shown in table 1, BMP7 administration significantly decreased the mortality rate after CVB3 injection (40\% vs. 25\%, $p<0.05)$. Additionally, the ratio of BMP7/TGF- $\beta 1$ in mRNA levels was demonstrated to decline obviously at different time points after CVB3 injection (Figure 1), denoting that CVB3 infection may contribute to the imbalance of BMP7/TGF- $\beta 1$ in the heart.

To assess the myocardial function, echocardiography was performed. The data revealed that CVB3 infection resulted in the development of a progressive left ventricular (LV) function deterioration reflected by the rise of the LVEDd and LVEDs and the decrease of LVEF and FS. However, BMP7 treatment significantly improved cardiac function, as characterized by the alteration of the echocardiographic functional parameters (Table 1, Figure 2).

To evaluate the extent of myocardial damage, the hearts were examined using HE and SR staining. The analysis revealed extensive myocardial damage and interstitial fibrosis in the hearts of VMC group, whereas BMP7 ameliorated myocardial necrosis and fibrosis (Figure 3A). In addition, murine heart weight to body weight ratio (HW/BW) was markely elevated in VMC group. However, BMP7 treatment decreased the HW/BW ratio compared with VMC group (Figure 3B). More than that, virus RNA replication was obviously decreased in CVB3-infected hearts by BMP7 administration (Figure 3C).

\subsection{BMP7 inhibited Endo-MT in viral myocarditis}

Compared with control group, immunohistochemistry staining showed that the expression of CD31, the marker of endothelial cells, was significantly decreased in VMC mice, which was attenuated by BMP7 (Figure 4, $P<0.05$ ). It was also found that FSP-1, a mesenchymal marker, was markedly increased in VMC mice, which was inhibited by BMP7 intervention (Figure 4, $p<0.01$ ). Those results suggested that Endo-MT was involved in CVB3-induced VMC.

To further delineate Endo-MT, co-expression of endothelial markers (CD31 and VE-cadherin, green) and mesenchymal markers (FSP-1-and aSMA, red) were analyzed using double 
immunofluorescence staining. Confocal microscopy revealed double-labeled of $\alpha \mathrm{SMA} / \mathrm{CD} 31$ and FSP-1/VE-cadherin after CVB3 intrusion, whereas the number of co-expression cells was greatly decreased with BMP7 intervention (Figure5). Based on these results, it was indicated that BMP7 inhibited Endo-MT in viral myocarditis, which might contribute to a switch to cardiac fibrosis (Figure 3A).

\subsection{BPM7 inhibited Smads and Wnt pathway}

TGF- $\beta 1 /$ Smads signaling pathway has been discovered to facilitate the pathogenesis of cardiac fibrosis, mainly by stimulating the collagen-producing cells [16, 17]. Additionally, it was demonstrated that canonical $\mathrm{Wnt} / \beta$-catenin signaling pathway was involved in epithelia-tomesenchymal transition (EMT) during development of embryonic atrioventricular cardiac cushion and heart disease[18-20]. Moreover, emerging studies have been showed that $\mathrm{Wnt} / \beta$-catenin signaling was interacted with TGF- $\beta$-mediated fibrosis[21]. Therefore, we evaluated both the TGF$\beta$ and Wnt signaling pathways in order to depict the molecular pathways involved in Endo-MT during viral myocardial fibrosis.

The TGF- $\beta$ signals through pSmad2/3, whereas the BMP7 signals through pSmad1/5/8 proteins. Smad4, a common partner, translocates to the nucleus to regulate the transcription of target genes[22]. In the present study, western blot analysis demonstrated that protein levels of pSmad3 and Smad4 were significantly upregulated in VMC group, as well as Wnt/ $\beta$-catenin and the transcription factor snail. BMP7 treatment reversed the changes of these protein levels, indicating that BMP7 may inhibit Endo-MT via TGF- $\beta /$ Smad and Wnt/ $\beta$-catenin pathway (Figure 6). Furthermore, the crosstalk between Smad3 and $\beta$-catenin was determined. Co-IP assay demonstrated that co-localization of $\beta$-catenin and Smad3 was increased in CVB3-infected mice. However, BMP7 treatment inhibited the protein complex between Smad3 and $\beta$-catenin (Figure 7). These results indicated that BMP7 hampered the activation of TGF- $\beta /$ Smad and Wnt/ $\beta$-catenin signaling after virus intrusion, which is associated with the interaction of Smad3/ $\beta$-catenin and upregulation of snail (Figure 8).

\section{Discussion}

The prominent findings of the present study demonstrated that BMP7 facilitated the recovery of cardiac function after CVB3 infection via inhibition of myocardial damage, collagen deposition, 
Endo-MT. Furthermore, myocardial fibrosis was impeded by BMP7 through inhibition of Endo-MT, which might be involved in the TGF- $\beta 1 / \mathrm{Smad} 3$ and $\mathrm{Wnt} / \beta$-catenin signaling.

Previous findings have demonstrated the emergence of cardiac fibroblasts with an endothelial cell origin through Endo-MT in DCM patients[5]. One-third of the myofibroblasts was demonstrated to transdifferentiate from cells of endothelial origin in fibrotic heart[6, 7, 23-25]. CVB3 infection induced Endo-MT in CMVECs in vitro[11]. Furthermore, the present study depicted that CVB3 stimulated Endo-MT and promote myocardial fibrosis in mouse models of myocarditis. These results indicated that Endo-MT may play a crucial role in the pathologic transformation from VMC to DCM.

TGF- $\beta 1$ is the primary stimulator of Endo-MT driving fibrosis in many different organs[2628]. On the other hand, BMP-7 is emerged as an antifibrotic cytokine in pathological organ fibrosis via inhibition of Endo-MT[29]. Previous results demonstrated that TGF- $\beta 1$ was significantly increased in CVB3-infected CMVECs[11] and in CVB3-induced myocarditis mice[30]. The ratios TGF-Bs/BMP-7 was found to increase significantly in the left ventricular remodelling response in a mouse model of transverse aortic constriction[22]. Furthermore, the ratio of TGF - $\beta 1 /$ BMP-7 may contribute to the progression of the epithelial-mesenchymal transition (EMT) in rat liver fibrosis[31]. In the study, it was shown that BMP7/TGF- $\beta 1$ ratio in mRNA levels was significantly declined followed by CVB3 invasion. Altogether, these results give rise to the possibility that the imbalance of BMP7/TGF- $\beta 1$ may be the initiating mechanism of Endo-MT and its subsequent fibrosis elicited by CVB3.

TGF- $\beta 1$ activates several signaling pathways in EMT, including TGF- $\beta /$ Smads signaling and Wnt signaling[32]. The TGF- $\beta$ signals through $\mathrm{pSmad} 2 / 3$, whereas the BMP7 signals through pSmad1/5/8 proteins. Smad3 is the direct mediator in TGF- $\beta 1$ signaling, which played a critical role in TGF- $\beta 1$-induced EMT and fibrosis[33]. Smad4 translocates to the nucleus to regulate the transcription of target genes[22]. Furthermore, Wnt/ $\beta$-catenin signaling was found to be activated to trigger Endo-MT in experimental myocardial infarction mice and DCM patients[5, 34]. $\beta$-catenin was required for Endo-MT in heart cushion formation[35]. In the study, we found that the protein levels of pSmad3, Smad4 and Wnt were evidently elevated by CVB3, which were hampered by BMP7. These data indicated that the activation of TGF- $\beta 1 /$ Smads and Wnt/ $\beta$-catenin pathway may play a critical role in the progression of viral myocarditis. 
Increasing lines of evidence indicates a crosstalk between TGF- $\beta 1 / \mathrm{Smad} 3$ and $\mathrm{Wnt} / \beta$-catenin signaling pathway in vascular smooth muscle cell proliferation[36]. TGF $\beta$ and Wnt/ $\beta$-catenin signaling pathways are firmly intertwined[37]. Smad3 prevents $\beta$-catenin degradation and facilitates nuclear translocation of $\beta$-catenin in chondrocytes and lens[38, 39]. $\beta$-Catenin $/ \operatorname{Smad} 3$ interaction was found to regulates TGF- $\beta$-induced EMT in the lens[38]. Our findings demonstrated the convergence of $\beta$-catenin and Smad3 induced by CVB3, which was hampered by BMP7. These results indicated that $\beta$-Catenin/Smad3 interaction may be associated with Endo-MT in the development of viral myocardial fibrosis.

It is well established that the snail family of transcriptional repressors played a critical role in regulating EMT, including snaill and snail2 (also known as slug). Snail induced EMT by directly inhibiting the transcription of E-cadherin and was associated with decreased E-cadherin abundance [40]. In addition, previous studies showed that upregulation of snail expression resulted in a direct repression of the VE-cadherin promoter, which was crucial for endothelial cell-to-cell junctions [41]. Overexpression of snail was enough to induce Endo-MT[42]. In this study, protein level of snail was increased by CVB3 infection and was attenuated by BMP7, indicating an important role of snail in the progression of virus induced Endo-MT.

\section{Conclusion}

Our findings indicated that BMP7 obviously ameliorated myocardial fibrosis in CVB3-infected mice via Endo-MT, which was involved in the TGF- $\beta /$ Smad and Wnt/ $\beta$-catenin pathway. $\beta$ Catenin/Smad3 interaction may be associated with Endo-MT in the development of viral myocardial fibrosis. The value of BMP7 and Endo-MT as therapeutic target for the palliative treatment of viral myocarditis deserves further attention.

\section{Abbreviations}

Endo-MT: endothelial-to-mesenchymal transition; coxsackievirus B3: CVB3; bone morphogenetic protein 7: BMP7; VMC: viral myocarditis; DCM: dilated cardiomyopathy; CMVECs: cardiac microvascular endothelial cells; TGF- $\beta$ : transforming growth factor- $\beta$; EMT: epithelialmesenchymal transition;

\section{Acknowledgements}

Not applicable. 
Dr. Chen and Dr. Ding designed the experiment. Dr. Xie and Dr. Liao performed the experiments.

242 Yong Yu analyzed and interpreted the data. Dr. Xie drafted the manuscript and Dr. Liao edited this article. All authors read and approved the final manuscript.

This research was funded by National Natural Science Foundation of China (No. 81300166, No.

\section{Availability of data and materials}

All data generated or analyzed during this study are included in this article.

\section{Ethics approval and consent to participate}

This study was approved by Animal Experimentation Ethics Committee of Zhongshan Hospital, Fudan University, and all procedures were performed in accordance with the Guide for the Care and Use of Laboratory Animals and the Regulation of Animal Protection Committee.

\section{Consent for publication}

Not applicable.

\section{Competing interests}

The authors declare that they have no competing interests.

\section{$257 \quad$ Author details}

2581 Division of Nephrology, Zhongshan Hospital, Fudan University, Shanghai 200032, China

2592 Department of Cardiology, Zhongshan Hospital, Fudan University, Shanghai 200032, China

2603 Key Laboratory of Viral Heart Diseases, Ministry of Public Health, Shanghai Institute of Cardiovascular Diseases, Zhongshan Hospital, Fudan University, Shanghai 200032, China

\section{Reference:}

1. Esfandiarei M, McManus BM: Molecular biology and pathogenesis of viral myocarditis. Annu

2. Kawai C: From myocarditis to cardiomyopathy: mechanisms of inflammation and cell death: learning from the past for the future. Circulation 1999, 99(8):1091-1100.

3. Kania G, Blyszczuk P, Eriksson U: Mechanisms of cardiac fibrosis in inflammatory heart disease.

Trends Cardiovasc Med 2009, 19(8):247-252.

4. Chen P, Xie Y, Shen E, Li GG, Yu Y, Zhang CB, Yang Y, Zou Y, Ge J, Chen R et al: Astragaloside IV 
attenuates myocardial fibrosis by inhibiting TGF-beta1 signaling in coxsackievirus B3-induced cardiomyopathy. Eur J Pharmacol 2011, 658(2-3):168-174.

5. Xie Y, Liao J, Yu Y, Guo Q, Yang Y, Ge J, Chen H, Chen R: Endothelialtomesenchymal transition in human idiopathic dilated cardiomyopathy. Mol Med Rep 2018, 17(1):961-969.

6. Goumans MJ, van Zonneveld AJ, ten Dijke P: Transforming growth factor beta-induced endothelial-to-mesenchymal transition: a switch to cardiac fibrosis? Trends Cardiovasc Med 2008, 18(8):293-298.

7. Zeisberg EM, Tarnavski O, Zeisberg M, Dorfman AL, McMullen JR, Gustafsson E, Chandraker A, Yuan X, Pu WT, Roberts AB et al: Endothelial-to-mesenchymal transition contributes to cardiac fibrosis. Nat Med 2007, 13(8):952-961.

8. Aisagbonhi O, Rai M, Ryzhov S, Atria N, Feoktistov I, Hatzopoulos AK: Experimental myocardial infarction triggers canonical Wnt signaling and endothelial-to-mesenchymal transition. Dis Model Mech 2011, 4(4):469-483.

9. Widyantoro B, Emoto N, Nakayama K, Anggrahini DW, Adiarto S, Iwasa N, Yagi K, Miyagawa K, Rikitake $Y$, Suzuki T et al: Endothelial cell-derived endothelin-1 promotes cardiac fibrosis in diabetic hearts through stimulation of endothelial-to-mesenchymal transition. Circulation 2010, 121(22):2407-2418.

10. Ghosh AK, Bradham WS, Gleaves LA, De Taeye B, Murphy SB, Covington JW, Vaughan DE: Genetic deficiency of plasminogen activator inhibitor-1 promotes cardiac fibrosis in aged mice: involvement of constitutive transforming growth factor-beta signaling and endothelialto-mesenchymal transition. Circulation 2010, 122(12):1200-1209.

11. Xie Y, Liao J, Li M, Wang X, Yang Y, Ge J, Chen R, Chen H: Impaired cardiac microvascular endothelial cells function induced by Coxsackievirus B3 infection and its potential role in cardiac fibrosis. Virus Res 2012, 169(1):188-194.

12. Zhang H, Liu Y, Yan L, Du W, Zhang X, Zhang M, Chen H, Zhang Y, Zhou J, Sun H et al: Bone morphogenetic protein-7 inhibits endothelial-mesenchymal transition in pulmonary artery endothelial cell under hypoxia. J Cell Physiol 2018, 233(5):4077-4090.

13. Weiskirchen R, Meurer SK: BMP-7 counteracting TGF-beta1 activities in organ fibrosis. Front Biosci (Landmark Ed) 2013, 18:1407-1434.

14. Zeisberg M, Hanai J, Sugimoto H, Mammoto T, Charytan D, Strutz F, Kalluri R: BMP-7 counteracts TGF-beta1-induced epithelial-to-mesenchymal transition and reverses chronic renal injury. Nat Med 2003, 9(7):964-968.

15. Zeisberg M, Kalluri R: Reversal of experimental renal fibrosis by BMP7 provides insights into novel therapeutic strategies for chronic kidney disease. Pediatr Nephrol 2008, 23(9):13951398.

16. Khan R, Sheppard R: Fibrosis in heart disease: understanding the role of transforming growth factor-beta in cardiomyopathy, valvular disease and arrhythmia. Immunology 2006, 118(1):10-24.

17. Dobaczewski M, Chen W, Frangogiannis NG: Transforming growth factor (TGF)-beta signaling in cardiac remodeling. J Mol Cell Cardiol 2011, 51(4):600-606.

18. Person AD, Garriock RJ, Krieg PA, Runyan RB, Klewer SE: Frzb modulates Wnt-9a-mediated beta-catenin signaling during avian atrioventricular cardiac cushion development. Dev Biol 2005, 278(1):35-48.

19. Cohen ED, Tian Y, Morrisey EE: Wnt signaling: an essential regulator of cardiovascular 
differentiation, morphogenesis and progenitor self-renewal. Development 2008, 135(5):789798.

20. von Gise A, Pu WT: Endocardial and epicardial epithelial to mesenchymal transitions in heart development and disease. Circ Res 2012, 110(12):1628-1645.

21. Akhmetshina A, Palumbo K, Dees C, Bergmann C, Venalis P, Zerr P, Horn A, Kireva T, Beyer C, Zwerina $\mathrm{J}$ et al: Activation of canonical Wnt signalling is required for TGF-beta-mediated fibrosis. Nat Commun 2012, 3:735.

22. Merino D, Villar A, García R, Tramullas M, Ruiz L, Ribas C, Cabezudo S, Nistal J, Hurlé M: BMP7 attenuates left ventricular remodelling under pressure overload and facilitates reverse remodelling and functional recovery. Cardiovascular research 2016, 110(3):331-345.

23. AK G, WS B, LA G, B DT, SB M, JW C, DE V: Genetic deficiency of plasminogen activator inhibitor-1 promotes cardiac fibrosis in aged mice: involvement of constitutive transforming growth factor-beta signaling and endothelial-to-mesenchymal transition. Circulation 2010, 122(12):1200-1209.

24. $H$ Z, H H, Z L, J P, X J, T W, H C, L L, X Y, T S et al: Pigment epithelium-derived factor attenuates myocardial fibrosis via inhibiting Endothelial-to-Mesenchymal Transition in rats with acute myocardial infarction. Scientific reports 2017, 7:41932.

25. X L, H M, B R, QH L, W Z, P L, N L, ES L, Q W, DQ T et al: Irisin inhibits high glucose-induced endothelial-to-mesenchymal transition and exerts a dose-dependent bidirectional effect on diabetic cardiomyopathy. Journal of cellular and molecular medicine 2018, 22(2):808-822.

26. Frid MG, Kale VA, Stenmark KR: Mature vascular endothelium can give rise to smooth muscle cells via endothelial-mesenchymal transdifferentiation: in vitro analysis. Circ Res 2002, 90(11):1189-1196.

27. Ishisaki A, Hayashi H, Li AJ, Imamura T: Human umbilical vein endothelium-derived cells retain potential to differentiate into smooth muscle-like cells. J Biol Chem 2003, 278(2):1303-1309.

28. Krenning G, Moonen JR, van Luyn MJ, Harmsen MC: Vascular smooth muscle cells for use in vascular tissue engineering obtained by endothelial-to-mesenchymal transdifferentiation (EnMT) on collagen matrices. Biomaterials 2008, 29(27):3703-3711.

29. Sugi Y, Yamamura H, Okagawa $H$, Markwald RR: Bone morphogenetic protein-2 can mediate myocardial regulation of atrioventricular cushion mesenchymal cell formation in mice. Dev Biol 2004, 269(2):505-518.

30. Xie Y, Chen R, Zhang X, Chen P, Liu X, Xie Y, Yu Y, Yang Y, Zou Y, Ge J et al: The role of Th17 cells and regulatory T cells in Coxsackievirus B3-induced myocarditis. Virology 2011, 421(1):78-84.

31. $\mathrm{Bi} \mathrm{W}, \mathrm{Xu} \mathrm{G}, \mathrm{Lv} \mathrm{L}$, Yang C: The ratio of transforming growth factor- $\beta 1 /$ bone morphogenetic protein-7 in the progression of the epithelial-mesenchymal transition contributes to rat liver fibrosis. Genetics and molecular research : GMR 2014, 13(1):1005-1014.

32. Gonzalez D, Medici D: Signaling mechanisms of the epithelial-mesenchymal transition. Science signaling 2014, 7(344):re8.

33. Shi Y, Massague J: Mechanisms of TGF-beta signaling from cell membrane to the nucleus. Cell 2003, 113(6):685-700.

34. $\mathrm{O} A, M R, S R, N A, I F, A K H$ : Experimental myocardial infarction triggers canonical Wnt signaling and endothelial-to-mesenchymal transition. Disease models \& mechanisms 2011, 4(4):469-483.

35. Liebner S, Cattelino A, Gallini R, Rudini N, lurlaro M, Piccolo S, Dejana E: Beta-catenin is 
required for endothelial-mesenchymal transformation during heart cushion development in the mouse. J Cell Biol 2004, 166(3):359-367.

36. DiRenzo DM, Chaudhary MA, Shi X, Franco SR, Zent J, Wang K, Guo LW, Kent KC: A crosstalk between TGF-beta/Smad3 and Wnt/beta-catenin pathways promotes vascular smooth muscle cell proliferation. Cell Signal 2016, 28(5):498-505.

37. Labbé E, Lock L, Letamendia A, Gorska A, Gryfe R, Gallinger S, Moses H, Attisano L: Transcriptional cooperation between the transforming growth factor-beta and Wnt pathways in mammary and intestinal tumorigenesis. Cancer research 2007, 67(1):75-84.

38. Taiyab A, Holms J, West-Mays JA: beta-Catenin/Smad3 Interaction Regulates Transforming Growth Factor-beta-Induced Epithelial to Mesenchymal Transition in the Lens. Int J Mol Sci 2019, 20(9).

39. Zhang M, Wang M, Tan X, Li TF, Zhang YE, Chen D: Smad3 prevents beta-catenin degradation and facilitates beta-catenin nuclear translocation in chondrocytes. J Biol Chem 2010, 285(12):8703-8710.

40. Nieto M: The snail superfamily of zinc-finger transcription factors. Nature reviews Molecular cell biology 2002, 3(3):155-166.

41. Lopez D, Niu G, Huber P, Carter W: Tumor-induced upregulation of Twist, Snail, and Slug represses the activity of the human VE-cadherin promoter. Archives of biochemistry and biophysics 2009, 482:77-82.

42. Cano A, Pérez-Moreno M, Rodrigo I, Locascio A, Blanco M, del Barrio M, Portillo F, Nieto M: The transcription factor snail controls epithelial-mesenchymal transitions by repressing $\mathrm{E}$ cadherin expression. Nature cell biology 2000, 2(2):76-83. 
Figure 1: BMP7 to TGF- $\beta 1$ ratio was on decline trend in mRNA levels at different time points after CVB3 injection.

Figure 2: As echo demonstrated, impaired cardiac function with increased parameters of LVEDd and LVEDs and declined LVEF and FS was found in VMC group, which was attenuated by BMP7 administration. *VMC group vs Control group, $p<0.05 ;{ }^{\S} \mathrm{VMC}+\mathrm{BMP} 7$ group $v s$ VMC group, $p<0.05$

Figure 3: A. HE and Sirus Red staining of heart tissue of three groups; B. Murine heart weight to body weight ratio; C. Virus replication in cardiac samples after CVB3 injection. * VMC group vs Control group, $p<0.05 ;$ VMC+BMP7 group vs VMC group, $p<0.05$

Figure 4: Immunohistochemistry staining of CD31 and FSP-1 in each group. ${ }^{\#}$ VMC group vs Control group, $p<0.05 ;{ }^{\S} \mathrm{VMC}+\mathrm{BMP} 7$ group $v s \mathrm{VMC}$ group, $p<0.05$

Figure 5: Co-localization of endothelial markers and mesenchymal markers. Double immunofluorescence staining of CD31 and $\alpha$ SMA, and VE-cadherin and FSP1 was performed. The expression of CD31 and VE-cadherin were colored with green, and the expression of $\alpha \mathrm{SMA}$ and FSP1 were colored with red. Co-labelled of CD31 and $\alpha$ SMA was observed in cardiac endothelial cells of VMC mice, as well as VE-cadherin and FSP1.

Figure 6: Western blot demonstrated the upregulated protein levels of pSmad3/Smad3 and Smad4 in VMC group, as well as the elevated protein expression of Wnt, $\beta$-catenin and its transcription factor snail. However, those TGF- $\beta 1 /$ Smads and Wnt/ $\beta$-catenin signaling protein have been downregulated after BMP7 intervention. ${ }^{\#}$ VMC group vs Control group, $\mathrm{p}<0.05 ;{ }^{\S} \mathrm{VMC}+\mathrm{BMP} 7$ group vs VMC group, $\mathrm{p}<0.05$

Figure 7: Co-IP assay revealed the increased interaction of endogenous $\beta$-catenin and $\operatorname{smad} 3$ in CVB3-infected mice, which was disrupted by BMP7. 
415 Figure 8: Schematic diagram of the protective effect of BMP7 against Endo-MT and cardiac fibrosis. 
Figures

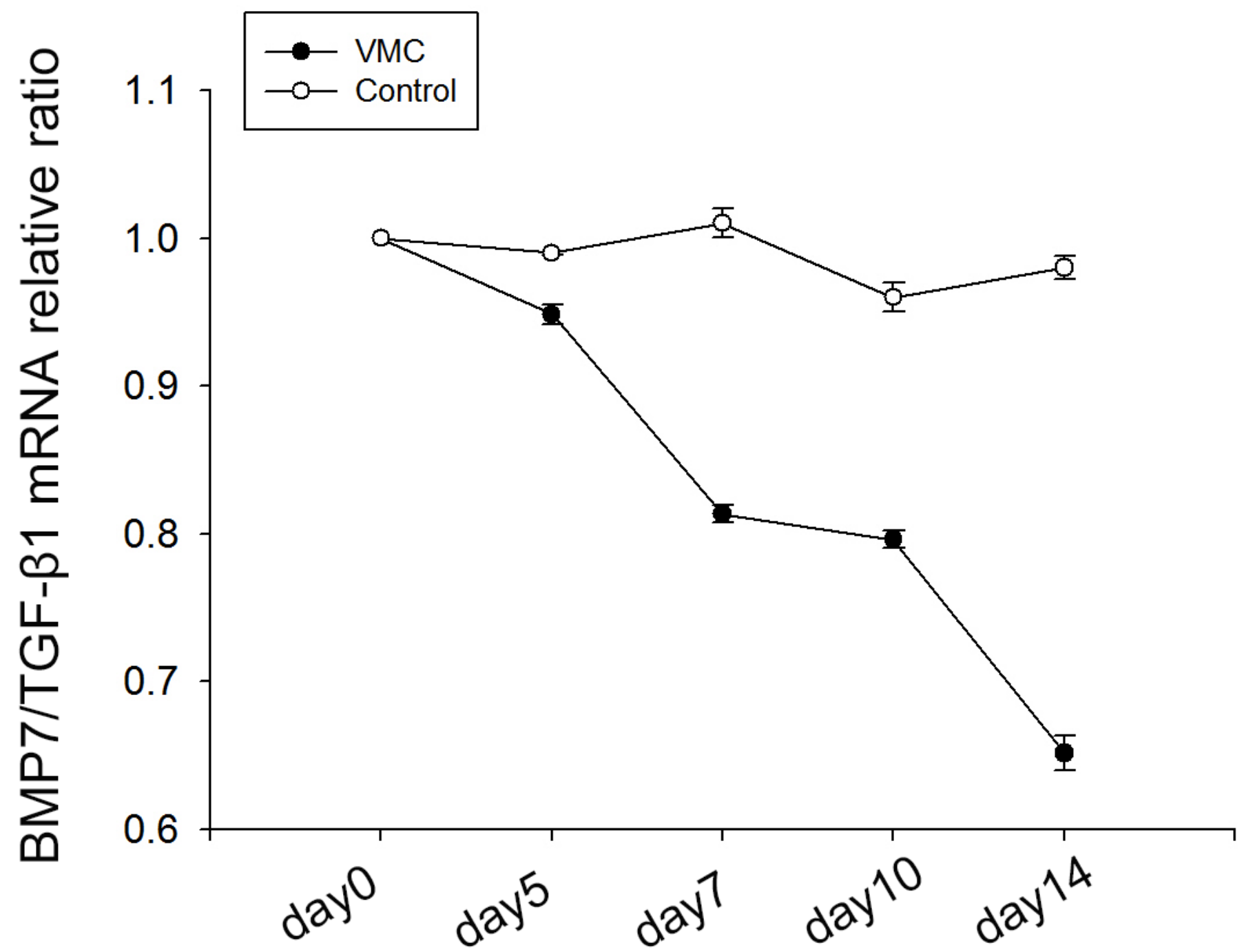

Figure 1

BMP7 to TGF- $\beta 1$ ratio was on decline trend in mRNA levels at different time points after CVB3 injection. 


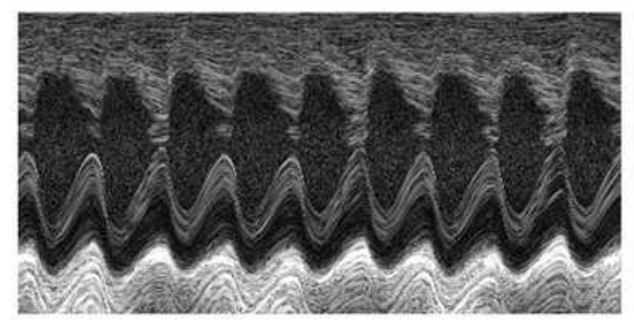

Control
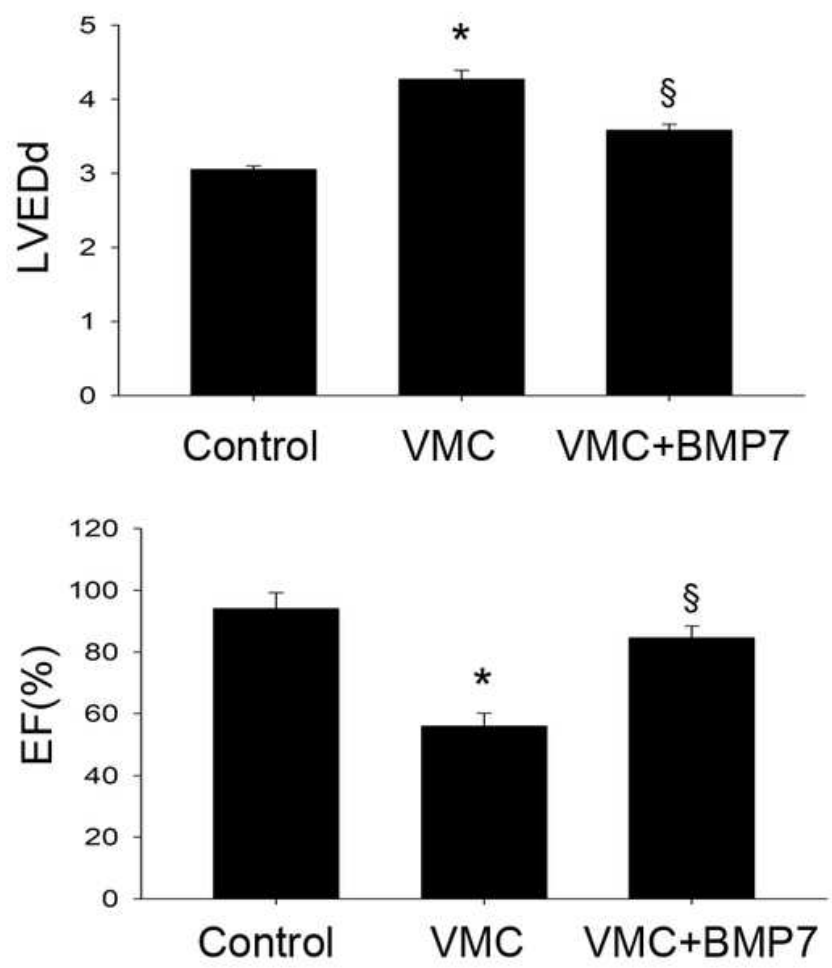

VMC
Figure 2

As echo demonstrated, impaired cardiac function with increased parameters of LVEDd and LVEDs and declined LVEF and FS was found in VMC group, which was attenuated by BMP7 administration. * VMC group vs Control group, $p<0.05 ; \S$ VMC+BMP7 group vs VMC group, $p<0.05$
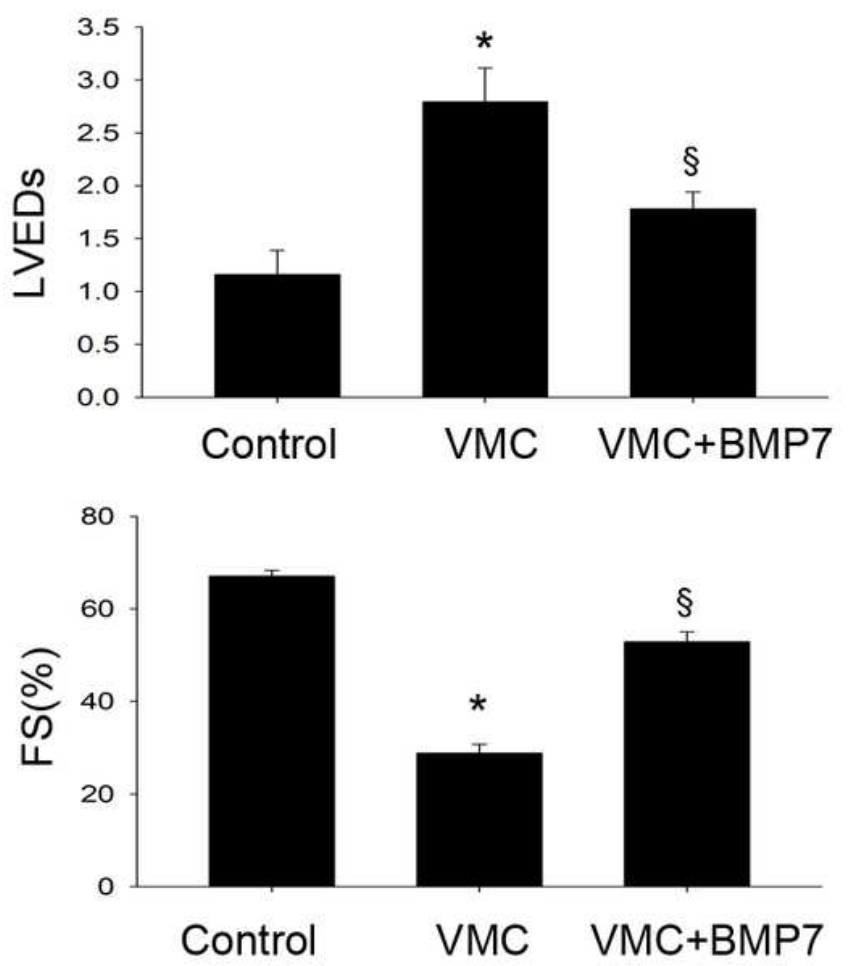
A
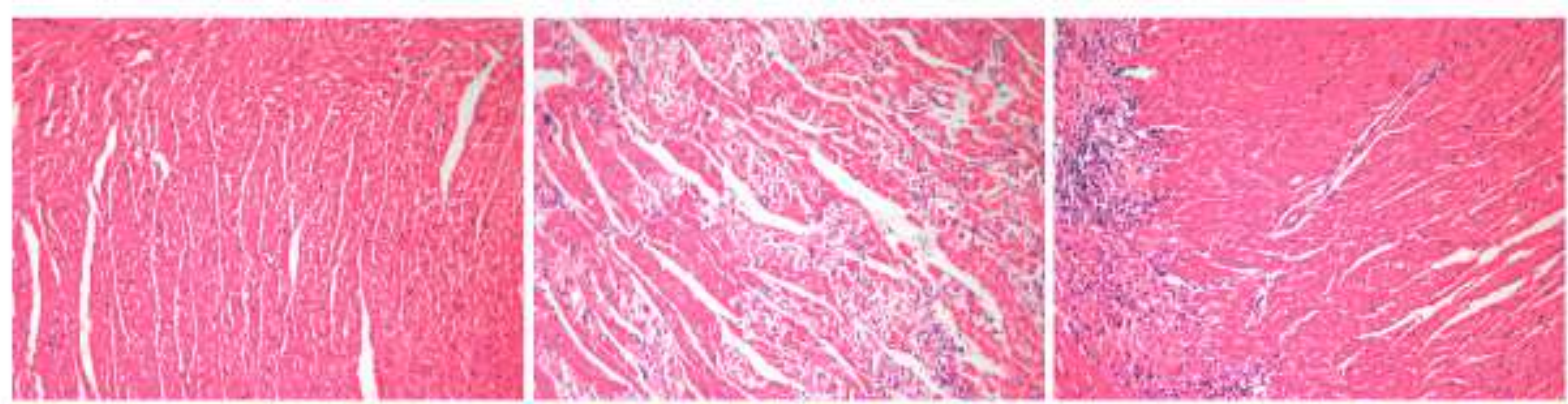

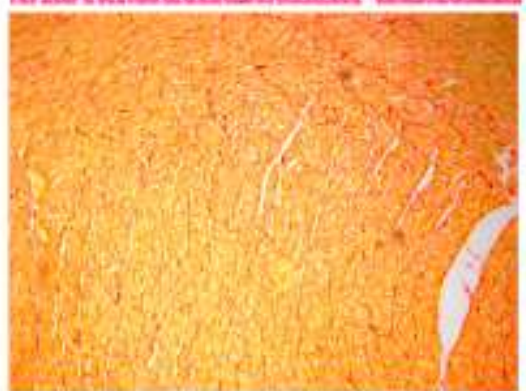

Control

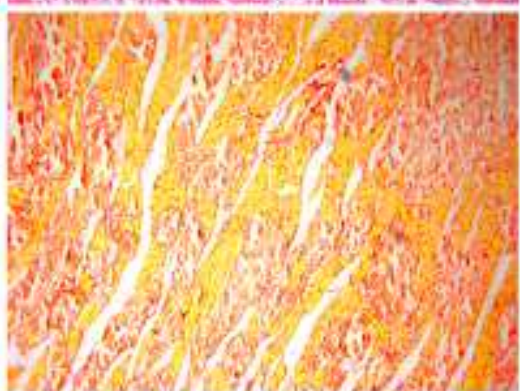

VMC

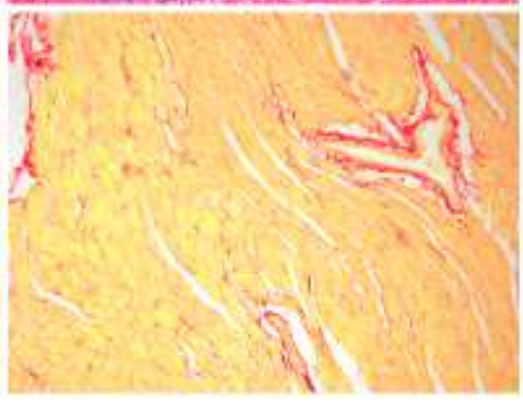

VMC+BMP7
B

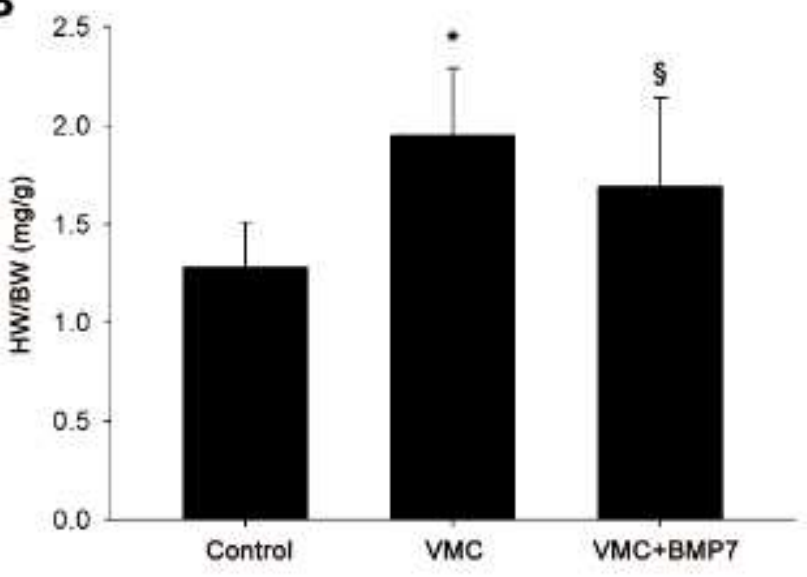

C

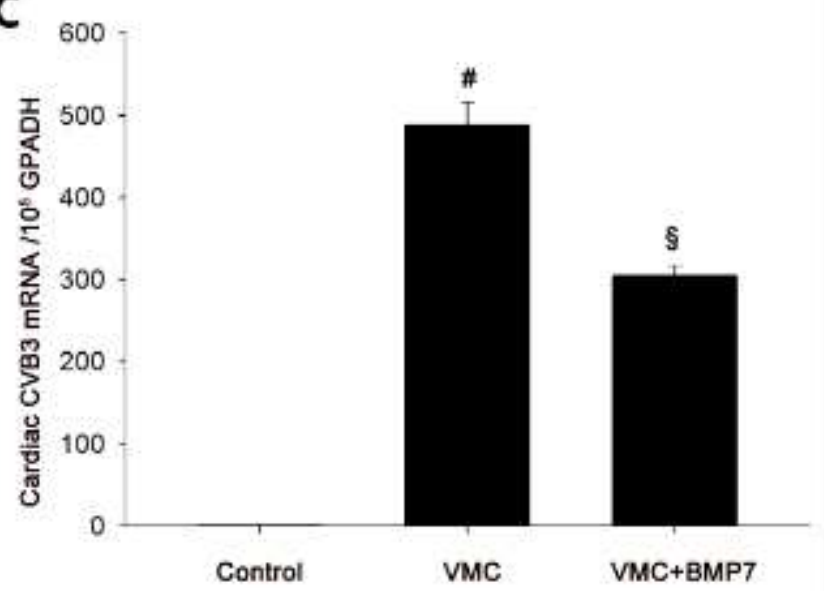

Figure 3

A. HE and Sirus Red staining of heart tissue of three groups; B. Murine heart weight to body weight ratio; C. Virus replication in cardiac samples after CVB3 injection. * VMC group vs Control group, $p<0.05$; $\S$ VMC+BMP7 group vs VMC group, $p<0.05$ 

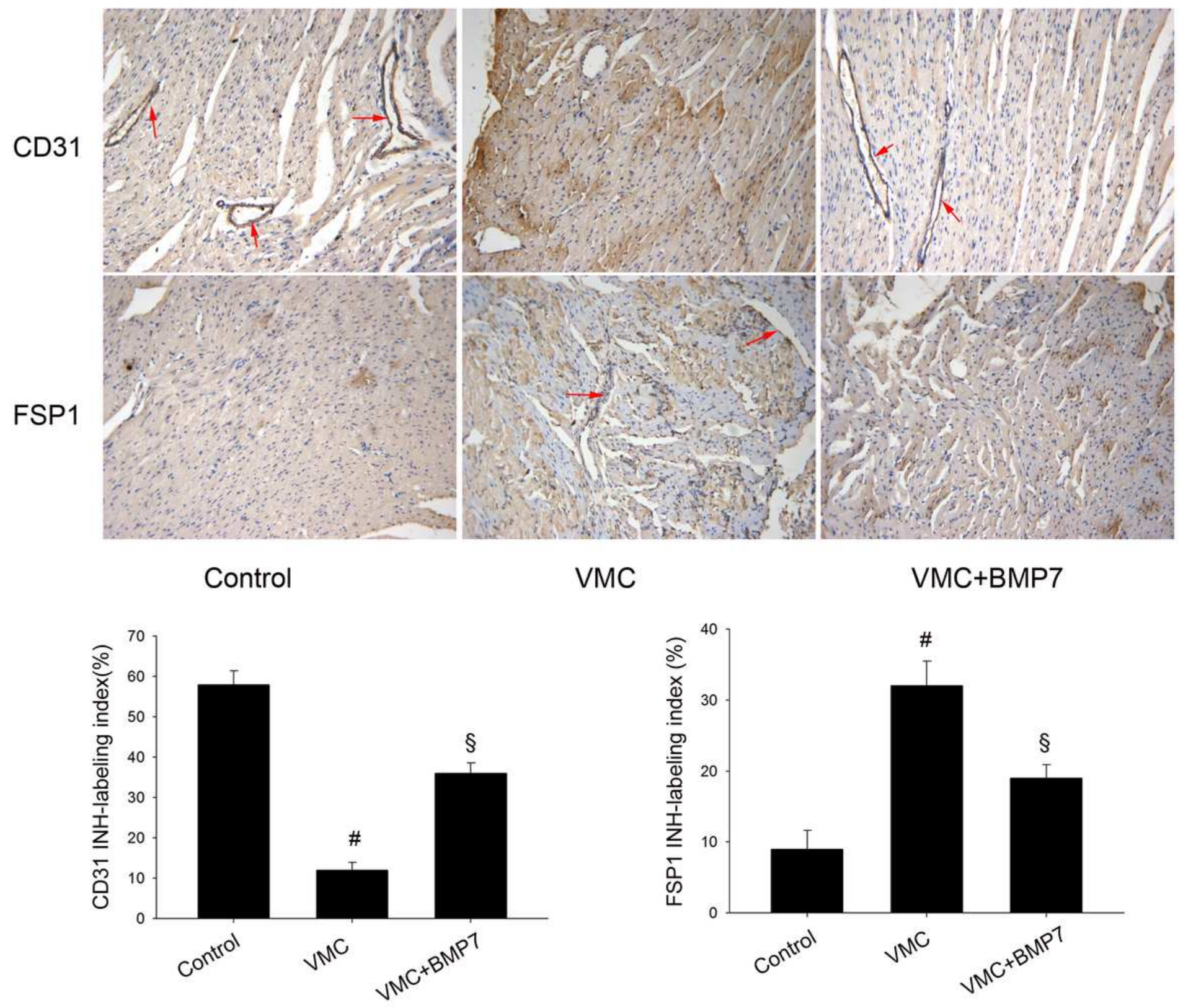

\section{Figure 4}

Immunohistochemistry staining of CD31 and FSP-1 in each group. \# VMC group vs Control group, $\mathrm{p}<0.05$; $\S$ VMC+BMP7 group vs VMC group, $p<0.05$ 

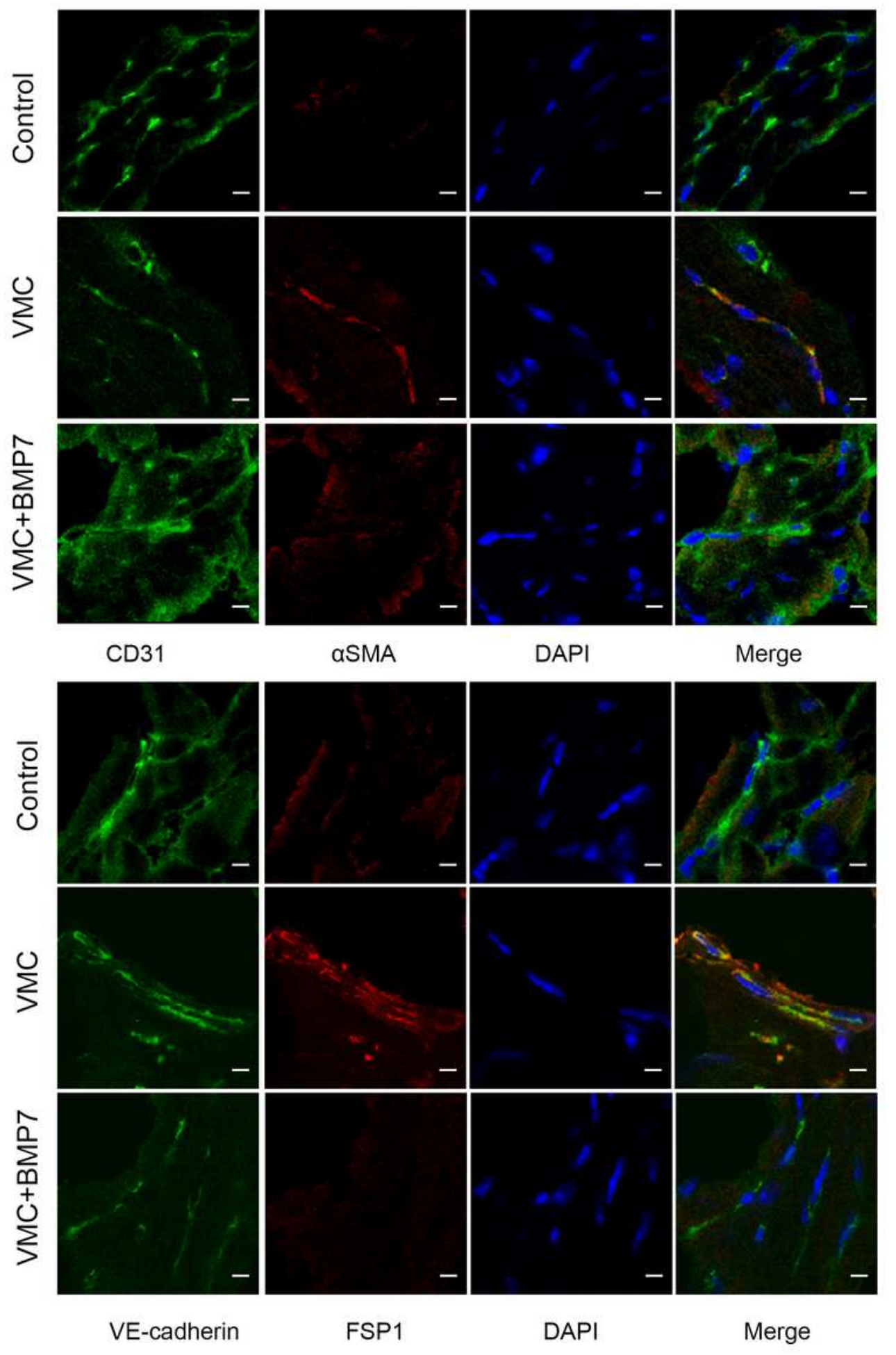

Figure 5

Co-localization of endothelial markers and mesenchymal markers. Double immunofluorescence staining of CD31 and aSMA, and VE-cadherin and FSP1 was performed. The expression of CD31 and VE-cadherin were colored with green, and the expression of aSMA and FSP1 were colored with red. Co-labelled of CD31 and aSMA was observed in cardiac endothelial cells of VMC mice, as well as VE-cadherin and FSP1. 
A

p-smad3

smad3

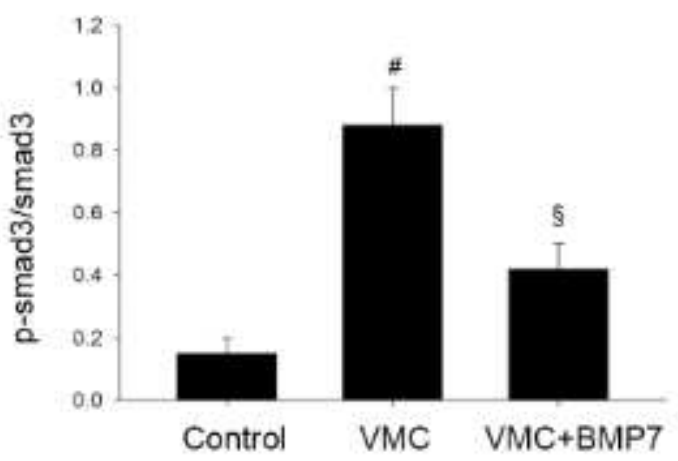

C
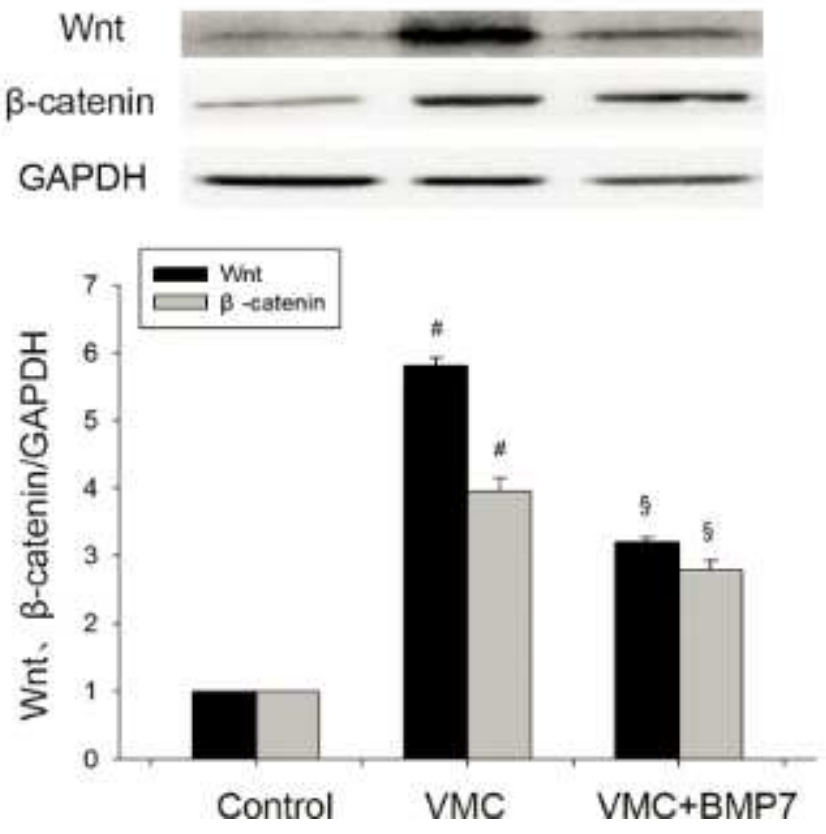

B

smad4

GAPDH

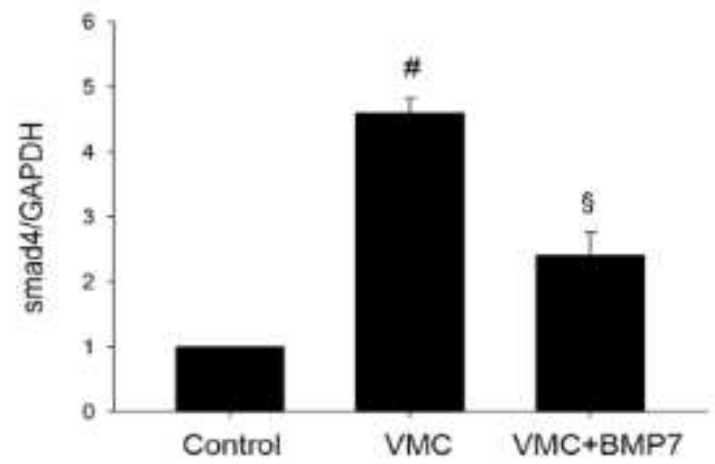

D
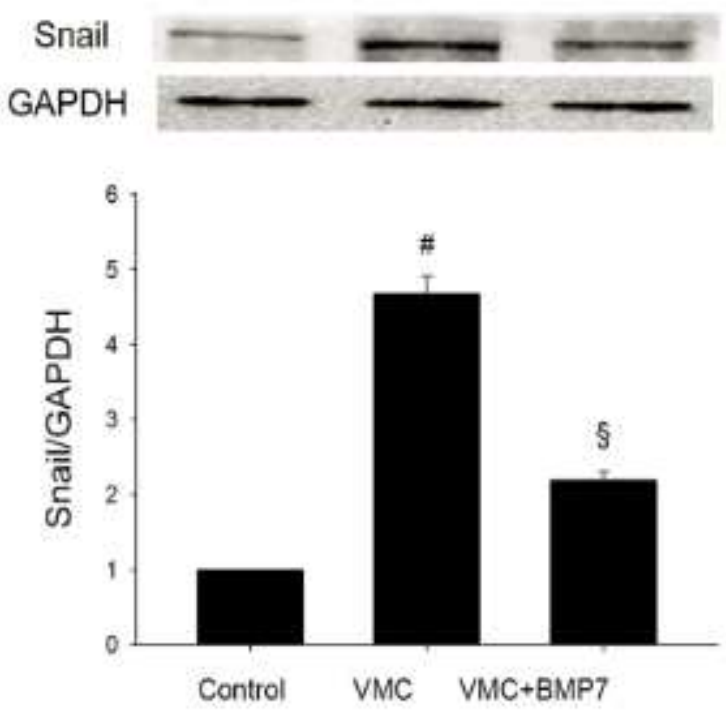

Figure 6

Western blot demonstrated the upregulated protein levels of pSmad3/Smad3 and Smad4 in VMC group, as well as the elevated protein expression of Wnt, $\beta$-catenin and its transcription factor snail. However, those TGF- $\beta 1 /$ Smads and Wnt/ $\beta$-catenin signaling protein have been downregulated after BMP7 intervention. \# VMC group vs Control group, $p<0.05$; $§$ VMC+BMP7 group vs VMC group, $p<0.05$ 

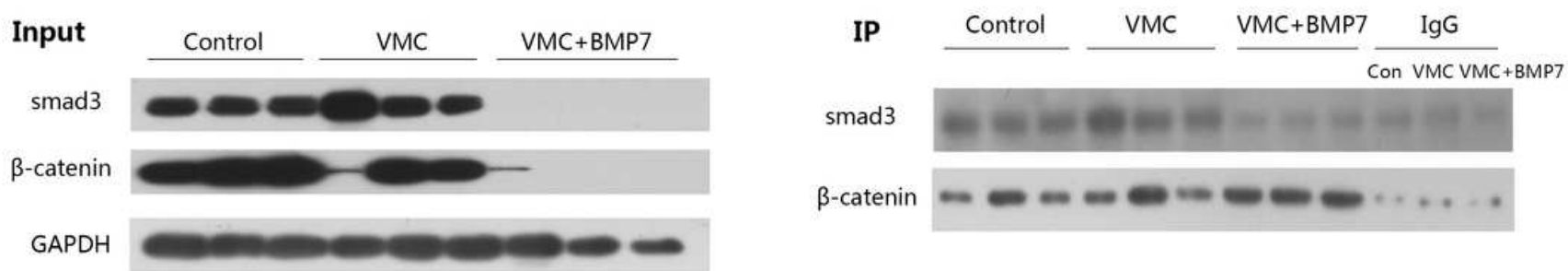

\section{Figure 7}

Co-IP assay revealed the increased interaction of endogenous $\beta$-catenin and smad3 in CVB3-infected mice, which was disrupted by BMP7. 


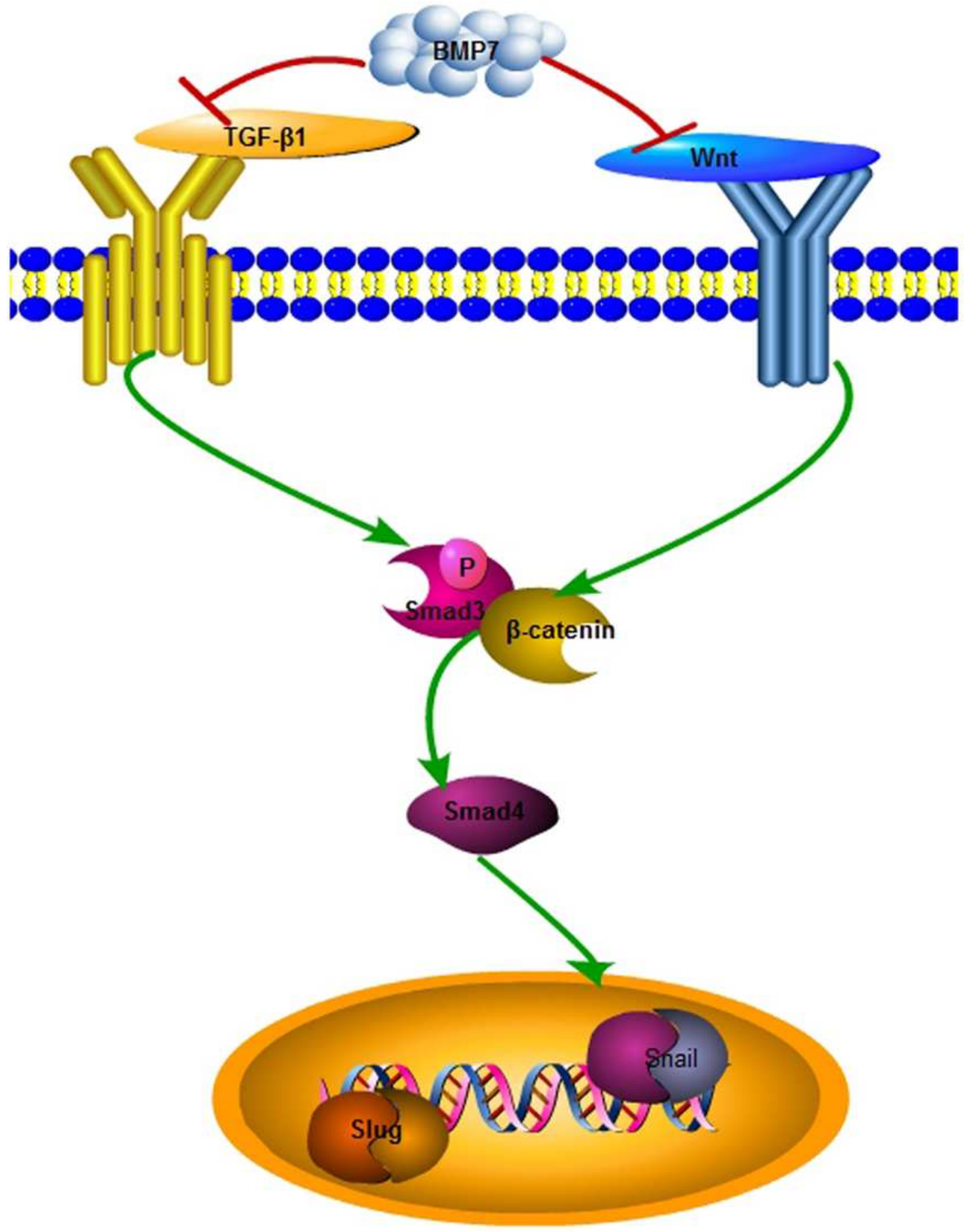

Figure 8

Schematic diagram of the protective effect of BMP7 against Endo-MT and cardiac fibrosis. 